\title{
Existence and uniqueness for the Prandtl equations
}

\section{Marco CANNONE $^{\text {a }}$, Maria Carmela LOMBARDO ${ }^{\text {b }}$, Marco SAMMARTINO ${ }^{\text {b }}$}

a Équipe d'analyse et de mathématiques appliquées, UFR de mathématiques, université de Marne-la-Vallée, cité Descartes, 5, boulevard Descartes, Champs-sur-Marne, 77454 Marne-la-Vallée cedex 2, France E-mail: cannone@math.univ-mlv.fr

${ }^{\text {b }}$ Dipartimento di Matematica, Università di Palermo, Via Archirafi 34, 90123 Palermo, Italy E-mail: lombardo@dipmat.math.unipa.it,marco@dipmat.math.unipa.it

(Reçu le 8 novembre 2000, accepté le 4 décembre 2000)

\begin{abstract}
Under the hypothesis of analyticity of the data with respect to the tangential variable we prove the existence and uniqueness of the mild solution of Prandtl boundary layer equation. This can be considered an improvement of the results of [8] as we do not require analyticity with respect to the normal variable. @ 2001 Académie des sciences/Éditions scientifiques et médicales Elsevier SAS
\end{abstract}

\section{Existence et unicité des équations de Prandtl}

Résumé. En supposant l'analyticité des données par rapport à la variable tangentielle nous prouvons l'existence et l'unicité de la solution des équations de Prandtl dans le demi-espace. Ce résultat peut être considéré comme une amélioration des résultats obtenus dans [8] : nous ne demandons pas l'analyticité des données par rapport à la variable normale. ( 2001 Académie des sciences/Éditions scientifiques et médicales Elsevier SAS

\section{Version française abrégée}

Dans cette Note nous considérons les équations de Prandtl dans le demi-espace défini par les équations (1.1)-(1.7). Ici, $\left(u^{\mathrm{P}}, v^{\mathrm{P}}\right)$ et $p^{\mathrm{P}}$ sont respectivement les composantes de la vitesse et de la pression du fluide à l'intérieur de la couche limite (BL). L'équation (1.5) permet d'établir le lien entre les équations BL et l'équation d'Euler à l'extérieur de la couche limite; $U(x, t)$ est la composante tangentielle de la solution de l'équation d'Euler aux bords; $x=\left(x_{1}, x_{2}\right)$ est la variable tangentielle et $Y$ la variable normale.

Les équations de Prandtl sont alors obtenues en prenant la limite des équations de Navier-Stokes quand la viscosité tend vers zéro et en supposant que, près du bord, le tenseur de viscosité est du même ordre que les forces d'inertie.

Malgré les progrès remarquables, obtenus dans la théorie des couches limites, nombreuses sont les questions fondamentales non encore résolues. Par exemple, on ne sait toujours pas si le système (1.1)-(1.7) est bien posé au sens d'Hadamard. Dans cette direction on citera les travaux d'Oleinik et de ses collaborateurs dans lesquels l'existence et l'unicité d'une solution des équations (1.1)-(1.7) sont prouvées

\section{Note présentée par Carlo CERCIGNANI.}


sous des hypothèses de monotonicité du flux $U$ et des données initiales $u_{\text {in }}^{\mathrm{P}}$. Pour une discussion et une bibliographie complètes sur ces questions nous renvoyons le lecteur à [6].

Sammartino et Caflisch ont démontré, dans [8], l'existence et l'unicité de la solution du système d'équations (1.1)-(1.7) sous l'hypothèse d'analyticité des données initiales par rapports aux deux variables $x$ et $Y$. Leur démonstration est essentiellement basée sur une version abstraite du théorème de Cauchy-Kowalewski (ACK), dans la formulation proposée par Safonov dans [7]. Le théorème ACK donne alors l'existence et l'unicité de la solution d'un problème aux valeurs initiales du premier ordre, à condition que le terme de forcing soit presque contractif. L'hypothèse d'analyticité est essentielle dans le contrôle des normes des termes contenant les dérivées en espace.

Dans cette Note nous généralisons les résultats de [8] et prouver l'existence et l'unicité de $u^{\mathrm{P}}$, en supposant l'analyticité des données par rapport à la variable tangentielle $x$. Ce qui est possible grâce à l'action de dissipation par rapport à la variable normale dans l'équation (1.1). Après avoir inversé l'opérateur parabolique, qui contient les $Y$-dérivées du premier ordre, nous allons résoudre par la méthode itérative l'équation correspondante. L'existence et l'unicité de la solution sont alors garanties en utilisant une version modifiée du théorème ACK.

Les résultats annoncés dans cette Note sont vrais en trois ou deux dimensions. Pour simplifier la présentation nous allons restreindre notre attention au cas bidimensionel.

\section{Introduction}

In this Note we shall be concerned with the Prandtl equations in the half space:

$$
\begin{array}{r}
\left(\partial_{t}-\partial_{Y Y}\right) u^{\mathrm{P}}+u^{\mathrm{P}} \partial_{x} u^{\mathrm{P}}+v^{\mathrm{P}} \partial_{Y} u^{\mathrm{P}}+\partial_{x} p^{\mathrm{P}}=0, \\
\partial_{Y} p^{\mathrm{P}}=0, \\
\partial_{x} u^{\mathrm{P}}+\partial_{Y} v^{\mathrm{P}}=0, \\
u^{\mathrm{P}}(x, Y=0, t)=v^{\mathrm{P}}(x, Y=0, t)=0, \\
u^{\mathrm{P}}(x, Y \rightarrow \infty, t) \longrightarrow U(x, t), \\
p^{\mathrm{P}}(x, Y \rightarrow \infty, t) \longrightarrow p^{E}(x, y=0, t), \\
u^{\mathrm{P}}(x, Y, t=0)=u_{\text {in }}^{\mathrm{P}} .
\end{array}
$$

In the above equations $\left(u^{\mathrm{P}}, v^{\mathrm{P}}\right)$ and $p^{\mathrm{P}}$ represent the components of the fluid velocity and the pressure inside the boundary layer (BL). Equation (1.5) is the matching condition between the flow inside the BL and the outer Euler flow; $U(x, t)$ is the tangential component of the Euler flow at the boundary; $x=\left(x_{1}, x_{2}\right)$ is the tangential variable, $Y$ the normal variable.

The Prandtl equations are obtained, in the limit of vanishing viscosity, from the Navier-Stokes equations assuming, close to the boundary, the viscous stresses to be of the same order of magnitude as the inertial forces.

In spite of the remarkably successful achievements of the boundary layer theory, many fundamental questions still remain unsolved. For instance the well-posedness of the system (1.1)-(1.7) has not yet been proved. Results have been obtained by Oleinik and coworkers. They proved the existence and the uniqueness of the solution of (1.1)-(1.7), under the hypotheses of the monotonicity of the outer flow $U$ and of the initial data $u_{\mathrm{in}}^{\mathrm{P}}$. For a complete and exhaustive review of their results see [6].

Sammartino and Caflisch in [8] proved the existence and the uniqueness of the Prandtl system, equations (1.1)-(1.7), under the hypothesis of analytic initial data with respect to both the spatial variables. The main ingredient of their proof was the abstract formulation of the Cauchy-Kowalewski theorem (ACK), in the form proposed by Safonov in [7]. The ACK theorem gives the existence and the uniqueness of the solution 
of a first order initial value problem, provided that the forcing term is shown to be almost contractive. The requirement of analyticity was crucial to control the norm of the terms containing the spatial derivatives.

In this Note we extend the result of [8], proving the existence and the uniqueness of $u^{\mathrm{P}}$, assuming analyticity of the initial data only with respect to the tangential variable $x$. This is made possible by the dissipativity with respect to the normal variable in equation (1.1). From a physical point of view, the assumption of analyticity with respect to the tangential variable has to be imposed to prevent the exponential growth of the $x$-disturbances whose length scale is $\mathrm{O}(\sqrt{\nu})$. We thus invert the parabolic operator, which includes the first order $Y$-derivative terms, and solve iteratively the resulting equation. The existence and uniqueness of the solution is then proved using a modified form of the ACK theorem.

These results are valid in 3-D and 2-D. To simplify the notation we shall restrict to the 2-D case.

In Section 2 we introduce the function spaces whilst in Section 3 we explicitly invert the parabolic operator appearing in equation (1.1), taking into account the first order $Y$-derivative, estimates of the solution in the proper function spaces are also given. The inverse parabolic operator is then used in Section 4 to put the Prandtl equation in a suitable form for the application of the ACK theorem. Finally, in Section 5 the ACK theorem is stated and applied to the mild form of (1.1)-(1.7) (see (4.1) below), so that the existence and the uniqueness of the solution are proved.

\section{Function spaces}

In this section we introduce the function spaces used in the proof of existence and uniqueness of the Prandtl equations. We first define the domain of analyticity with respect to the tangential variable $D(\rho)=\{x \in \mathbb{C}: \Im \mathrm{m} x \in(-\rho, \rho)\}$.

We now introduce the ambient spaces for the Prandtl equations.

Definition 2.1. - The space $K^{\ell, \rho}$ is the space of the functions $f(x)$ such that: (i) $f$ is analytic in $D(\rho)$; (ii) if $\Im \mathrm{m} x \in(-\rho, \rho)$ and $0 \leqslant j \leqslant \ell$, then $\partial_{x}^{j} f(\Re \mathrm{e} x+i \Im \mathrm{m} x)$ is square integrable in $\Re \mathrm{e} x$; (iii) $|f|_{\ell, \rho} \equiv \sum_{j=0}^{\ell} \sup _{\Im \mathrm{m} x \in(-\rho, \rho)}\left\|\partial_{x}^{j} f(\cdot+i \Im \mathrm{m} x)\right\|_{\mathrm{L}^{2}(\Re \mathrm{e} x)}<\infty$.

DEFinition 2.2. - The space $K^{\ell, \rho, \mu}$, with $\mu>0$, is the space of the functions $f(Y, x)$ such that $\mathrm{e}^{\mu Y} \partial_{x}^{i} \partial_{Y}^{j} f \in \mathrm{L}^{\infty}\left(\mathbb{R}^{+}, K^{0, \rho}\right)$ when $i+j \leqslant \ell$ and $j \leqslant 2$;

The norm in $K^{\ell, \rho, \mu}$ is defined as $|f|_{\ell, \rho, \mu} \equiv \sum_{j \leqslant 2} \sum_{i \leqslant \ell-j} \sup _{Y \in \mathbb{R}^{+}} \mathrm{e}^{\mu Y}\left|\partial_{Y}^{j} \partial_{x}^{i} f(Y, \cdot)\right|_{0, \rho}$

DEFINITION 2.3. - The space $K_{\beta, T}^{\ell, \rho}$, with $\beta>0$, is the space of the functions $f(x, t)$ such that $\partial_{t}^{i} \partial_{x}^{j} f(x, t) \in K^{\ell, \rho-\beta t}$, for all $0 \leqslant t \leqslant T$, where $0 \leqslant i+j \leqslant \ell$ and $0 \leqslant i \leqslant 1$.

Moreover, $|f|_{\ell, \rho, \beta, T} \equiv \sum_{0 \leqslant j \leqslant 1} \sum_{i \leqslant \ell-j} \sup _{0 \leqslant t \leqslant T}\left|\partial_{t}^{j} \partial_{x}^{i} f(\cdot, t)\right|_{0, \rho-\beta t}<\infty$.

DEFinition 2.4. - The space $K_{\beta, T}^{\ell, \rho, \mu}$ is the space of the functions $f(x, Y, t)$ such that $f \in K^{\ell, \rho-\beta t, \mu-\beta t}$ and $\partial_{t} \partial_{x}^{i} f \in K^{0, \rho-\beta t, \mu-\beta t}$ for all $0 \leqslant t \leqslant T$, where $0 \leqslant i \leqslant \ell-2$. Moreover,

$$
|f|_{\ell, \rho, \mu, \beta, T} \equiv \sum_{j=0}^{2} \sum_{i \leqslant \ell-j} \sup _{0 \leqslant t \leqslant T}\left|\partial_{Y}^{j} \partial_{x}^{i} f(\cdot, \cdot, t)\right|_{0, \rho-\beta t, \mu-\beta t}+\sum_{i \leqslant \ell-2} \sup _{0 \leqslant t \leqslant T}\left|\partial_{t} \partial_{x}^{i} f(\cdot, \cdot, t)\right|_{0, \rho-\beta t, \mu-\beta t}<\infty .
$$

\section{A convection-diffusion equation}

In this section we shall be concerned with the following equations:

$$
\begin{aligned}
\left(\partial_{t}-\partial_{Y Y}\right) u+\alpha Y \partial_{Y} u & =f, \\
u(x, Y=0, t) & =g, \\
u(x, Y, t=0) & =u_{0},
\end{aligned}
$$

where $\alpha$ is a function of $x$ and $t$. 
To solve the above system, we first introduce the following two kernels:

$$
\begin{aligned}
& F_{\alpha}(x, Y, t)=\frac{1}{\sqrt{4 \pi}} \frac{1}{\left(\int_{0}^{t} \mathrm{~d} \tau \mathrm{e}^{-2 A(x, \tau)}\right)^{1 / 2}} \exp \left(\frac{Y^{2} \mathrm{e}^{-2 A(x, t)}}{4\left(\int_{0}^{t} \mathrm{~d} \tau \mathrm{e}^{-2 A(x, \tau)}\right)}\right), \\
& H_{\alpha}(x, Y, t)=-2 \frac{\partial F_{\alpha}}{\partial Y}+2 \alpha Y F_{\alpha}(x, Y, t)-\alpha(x, t) E_{\alpha}(x, Y, t),
\end{aligned}
$$

where $A(\tau)$ and $E_{\alpha}$ are defined as:

$$
A(x, \tau)=\int_{0}^{\tau} \mathrm{d} \theta \alpha(x, \theta) \quad \text { and } \quad E_{\alpha}(x, Y, t)=\int_{0}^{\infty} \mathrm{d} Y^{\prime}\left[F_{\alpha}\left(x, Y-Y^{\prime}, t\right)-F_{\alpha}\left(x, Y+Y^{\prime}, t\right)\right] .
$$

We can introduce the following operators:

$$
\begin{aligned}
M_{0} u_{0} & =\int_{0}^{\infty} \mathrm{d} Y^{\prime}\left[F_{\alpha}\left(Y-Y^{\prime}, t\right)-F_{\alpha}\left(Y+Y^{\prime}, t\right)\right] u_{0}\left(x, Y^{\prime}\right), \\
M_{2} f & =\int_{0}^{t} \mathrm{~d} s \int_{0}^{\infty} \mathrm{d} Y^{\prime}\left[F_{\alpha}\left(Y-Y^{\prime}, t-s\right)-F_{\alpha}\left(Y+Y^{\prime}, t-s\right)\right] f\left(x, Y^{\prime}, s\right), \\
M_{1} g & =2 \int_{0}^{t} \mathrm{~d} s H_{\alpha}(Y, t-s) g(x, s) .
\end{aligned}
$$

The operator $M_{0}(t)$ is the convolution of the kernel $F_{\alpha}$ with the odd extension to $Y<0$ of the function $u_{0}(x, Y)$. It solves (3.1)-(3.3) with no source, homogeneous boundary data and with initial data. The operator $M_{2}$ solves (3.1)-(3.3) with homogeneous boundary and initial data and with source term. The operator $M_{1}$ solves (3.1)-(3.3) with zero source, zero initial data and with boundary data.

Using the explicit expressions of the operators $M_{0}, M_{1}$ and $M_{2}$ given by (3.5)-(3.7), one can write the explicit expression of the initial boundary value problem (3.1)-(3.3), and prove the following proposition:

PROPOSITION 3.1. - Suppose $\alpha, g \in K_{\beta, T}^{\ell, \rho}, f \in K_{\beta, T}^{\ell, \rho, \mu}$ and $u_{0} \in K^{\ell, \rho, \mu}$. Moreover let the compatibility condition $g(x, t=0)=u_{0}(x, Y=0)$ be satisfied. Then the solution $u$ of (3.1)-(3.3) is in $K_{\beta, T}^{\ell, \rho, \mu}$, and the following estimate holds: $|u|_{\ell, \rho, \mu, \beta, T} \leqslant c\left(|\alpha|_{\ell, \rho, \beta, T}+|f|_{\ell, \rho, \mu, \beta, T}+\left|u_{0}\right|_{\ell, \rho, \mu}+|g|_{\ell, \rho, \beta, T}\right)$.

We will also need the following estimates for the operator $M_{2}$ :

LEMma 3.1. - Suppose $\alpha \in K_{\beta, T}^{\ell, \rho}$ and $f \in K_{\beta, T}^{\ell, \rho, \mu}$ with $f(x, Y=0, t)=0$. If $\rho^{\prime}<\rho-\beta t$ and $\mu^{\prime}<\mu-\beta t$ then the following estimate holds: $\left|M_{2} f\right|_{\ell, \rho^{\prime}, \mu^{\prime}} \leqslant c \int_{0}^{t} \mathrm{~d} s|f(\cdot, \cdot, s)|_{\ell, \rho^{\prime}, \mu^{\prime}} \leqslant c|f|_{\ell, \rho, \mu, \beta, T}$.

Lemma 3.2. - Suppose $\alpha \in K_{\beta, T}^{\ell, \rho}, h \in K_{\beta, T}^{\ell, \rho, \mu},\left.\partial_{Y} h\right|_{Y=0} \in K_{\beta, T}^{\ell, \rho}$ with $0<\mu^{\prime}<\mu(s)<\mu-\beta s$. Then $M_{2} \partial_{Y} h \in K^{\ell, \rho, \mu^{\prime}}$ for each $0<t<T$ and the following estimate holds:

$$
\left|M_{2} \partial_{Y} h\right|_{\ell, \rho, \mu^{\prime}} \leqslant c \int_{0}^{t} \mathrm{~d} s\left(\frac{|h(\cdot, \cdot, s)|_{\ell, \rho, \mu^{\prime}}}{\sqrt{t-s}}+\frac{|h(\cdot, \cdot, s)|_{\ell, \rho, \mu(s)}}{\mu(s)-\mu^{\prime}}\right) .
$$

For the details of the proofs of the above statements see [4].

\section{The mild solutions of the Prandtl equations}

Following [8], we introduce the new variable $u=u^{P}-U$, so that, using the Euler equation at the boundary, (1.1)-(1.7) can be written in the form:

$$
u=F(u, t),
$$

where: 


$$
\begin{aligned}
& F(u, t)=M_{2} f+M_{0}(t) u_{0}-M_{1} U, \\
& f(u, t)=-u \partial_{x} u+\left(\int_{0}^{Y} \mathrm{~d} Y^{\prime} \partial_{x} u\right) \partial_{Y} u-U \partial_{x} u-u \partial_{x} U,
\end{aligned}
$$

and $M_{0}, M_{1}$ and $M_{2}$ are the operators defined in the previous section. We shall call the equation (4.1) the mild form of the Prandtl equations.

\section{The Cauchy estimate}

To prove the existence and the uniqueness of the mild solution of the Prandtl equations, we shall give a slightly modified version of the Abstract Cauchy-Kowalewski theorem (ACK) as given in [7] or [1] and [3].

For $t$ in $[0, T]$, consider the equation:

$$
u+F(t, u)=0 .
$$

Let $\left\{X_{\rho}: 0<\rho \leqslant \rho_{0}\right\}$ be a Banach scale with norms ||$_{\rho}$, such that $X_{\rho^{\prime}} \subset X_{\rho^{\prime \prime}}$ and ||$_{\rho^{\prime \prime}} \leqslant||_{\rho^{\prime}}$ when $\rho^{\prime \prime} \leqslant \rho^{\prime} \leqslant \rho_{0}$.

THEOREM 5.1 (ACK). - Suppose that there exists $R>0, T>0, \rho_{0}>0$, and $\beta_{0}>0$ such that if $0<t \leqslant T$, the following hold:

1. for all $0<\rho^{\prime}<\rho \leqslant \rho_{0}-\beta_{0} T$ and for all $u$ such that $\left\{u \in X_{\rho}: \sup _{0 \leqslant t \leqslant T}|u(t)|_{\rho} \leqslant R\right\}$ the function $F(t, u):[0, \tau] \mapsto X_{\rho^{\prime}}$ is continuous;

2. for all $0<\rho \leqslant \rho_{0}-\beta_{0} T$ the function $F(t, 0):[0, T] \mapsto\left\{u \in X_{\rho}: \sup _{0 \leqslant t \leqslant T}|u(t)|_{\rho} \leqslant R\right\}$ is continuous in $[0, T]$ and $|F(t, 0)|_{\rho_{0}-\beta_{0} t} \leqslant R_{0}<R$;

3. for all $0<\rho^{\prime}<\rho(s) \leqslant \rho_{0}-\beta_{0}$ s and for all $u^{1}$ and $u^{2} \in\left\{u \in X_{\rho}: \sup _{0 \leqslant t \leqslant T}|u(t)|_{\rho-\beta_{0} t} \leqslant R\right\}$,

$$
\left|F\left(t, u^{1}\right)-F\left(t, u^{2}\right)\right|_{\rho^{\prime}} \leqslant C \int_{0}^{t} \mathrm{~d} s\left(\frac{\left|u^{1}-u^{2}\right|_{\rho(s)}}{\rho(s)-\rho^{\prime}}+\frac{\left|u^{1}-u^{2}\right|_{\rho^{\prime}}}{\sqrt{t-s}}\right) .
$$

Then there exists $\beta>\beta_{0}$ and $0<T_{1}<T$ such that (5.1) has a unique solution $u(t) \in X_{\rho_{0}-\beta t}$ with $t \in\left[0, T_{1}\right] ;$ moreover, $\sup _{0 \leqslant t \leqslant T_{1}}|u(t)|_{\rho_{0}-\beta t} \leqslant R$.

For the proof of the above theorem (see [4]).

To apply the ACK theorem to the Prandtl equation, we have to prove that the right-hand side of (4.2) satisfies an estimate such as (5.2). We observe that, using Proposition 3.1, this is true for the first two terms.

The term $M_{2} f(u, x, Y, t)$ consists of four terms, namely:

$$
M_{2} f(u, x, Y, t)=-M_{2}\left(u \partial_{x} u\right)-M_{2}\left(U \partial_{x} u\right)-M_{2}\left(u \partial_{x} U\right)+M_{2}\left[\left(\int_{0}^{Y} \mathrm{~d} Y^{\prime} \partial_{x} u\right) \partial_{Y} u\right] .
$$

The first three terms in the above expression are easily estimated using Lemma 3.1 and through the following estimate, which is a consequence of the Cauchy estimate of the derivative of an analytic function:

Proposition 5.1. - Let $f \in K^{\ell, \rho^{\prime \prime}}$. If $\rho^{\prime}<\rho^{\prime \prime}$ then:

$$
\left|\partial_{x} f\right|_{\ell, \rho^{\prime}} \leqslant \frac{|f|_{\ell, \rho^{\prime \prime}}}{\rho^{\prime \prime}-\rho^{\prime}} .
$$

In order to estimate the term $M_{2}\left[\left(\int_{0}^{Y} \mathrm{~d} Y^{\prime} \partial_{x} u\right) \partial_{Y} u\right]$ we proceed as follows. Using integration by parts, we write it as:

$$
M_{2}\left[\left(\int_{0}^{Y} \mathrm{~d} Y^{\prime} \partial_{x} u\right) \partial_{Y} u\right]=M_{2}\left[\partial_{Y}\left(u \int_{0}^{Y} \mathrm{~d} Y^{\prime} \partial_{x} u\right)\right]-M_{2}\left(u \partial_{x} u\right) .
$$

The second term on the right-hand side of (5.4) is estimated through Lemma 3.1 and the Cauchy estimate, (5.3). The first term is estimated through Lemma 3.2 and the Cauchy estimate for the $x$-derivative inside the integral. 
Therefore the following proposition, giving an estimate of $F$, as defined in (4.2), has been proved:

Proposition 5.2. - Suppose that $u^{1}$ and $u^{2}$ are in $K_{\beta_{0}, T}^{\ell, \rho_{0}, \mu_{0}}$. Suppose $0<\rho^{\prime}<\rho(s)<\rho_{0}-\beta_{0}$ s and $0<\mu^{\prime}<\mu(s)<\mu_{0}-\beta_{0}$ s. Then the following estimate holds:

$$
\left|F\left(u^{1}, t\right)-F\left(u^{2}, t\right)\right|_{\ell, \rho^{\prime}, \mu^{\prime}} \leqslant c \int_{0}^{t} \mathrm{~d} s\left(\frac{\left|u^{1}-u^{2}\right|_{\ell, \rho(s), \mu}}{\rho(s)-\rho^{\prime}}+\frac{\left|u^{1}-u^{2}\right|_{\ell, \rho, \mu(s)}}{\mu(s)-\mu^{\prime}}+\frac{\left|u^{1}-u^{2}\right|_{\ell, \rho^{\prime}, \mu^{\prime}}}{\sqrt{t-s}}\right) .
$$

Therefore using the above proposition and the ACK theorem, the main result of this Note can be proved.

THEOREM 5.3. - Suppose $U \in K_{\beta_{0}, T}^{\ell, \rho_{0}}$ and $u_{\mathrm{in}}^{\mathrm{P}}-U \in K^{\ell, \rho_{0}, \mu_{0}}$. Moreover let the compatibility conditions $u_{\mathrm{in}}^{\mathrm{P}}(x, Y=0)=0, u_{\mathrm{in}}^{\mathrm{P}}(x, Y \rightarrow \infty)-U \rightarrow 0$ hold. Then there exists a unique mild solution $u^{\mathrm{P}}$ of (1.1)-(1.7). This solution can be written as $u^{\mathrm{P}}(x, Y, t)=u(x, Y, t)+U$, where $u \in K_{\beta_{1}, T_{1}}^{\ell, \rho_{1}, \mu_{1}}$ with $0<$ $\rho_{1}<\rho_{0}, 0<\mu_{1}<\mu_{0}, \beta_{1}>\beta_{0}>0$ and $0<T_{1}<T$.

Conclusions. - In this Note we have proved the existence and uniqueness of the boundary layer equations, requiring for the initial data analyticity with respect to the $x$ variable and $\mathrm{L}^{2}$-integrability with respect to the $Y$ variable. This extends the results of [8] and paves the way for future researches. For instance one could try to address the problem of the zero-viscosity limit of the Navier-Stokes equations in domains with curved boundaries. It should also be possible to give a further generalization of the hypotheses on the data of Prandtl equations; in fact the properties of the kernel $F_{\alpha}$ are quite similar to the properties of the heat kernel: this should allow us to introduce, for the Prandtl equations, the techniques and the ideas typical of the Besov spaces.

Nevertheless, a recent work (see [5]) seems to suggest that, if the Prandtl equations admit a solution in Sobolev spaces, such a solution is linearly and nonlinearly unstable and, therefore, it does not converge in $\mathrm{H}^{1}$ to the Navier-Stokes solution.

Many open problems within the boundary layer theory still remain, among which are the well-posedness of the equations, characterization of singularities and the mathematical theory of separation (for a review see [2]).

Acknowledgements. M.C. Lombardo and M. Sammartino are partially supported by the INDAM and the MURST respectively.

\section{References}

[1] Asano K., A note on the abstract Cauchy-Kowalewski theorem, Proc. Japan Acad. Ser. A 64 (A) (1988) 102-105.

[2] Caflisch R.E., Sammartino M., Existence and singularities for the Prandtl boundary layer equations, Z. Angew. Math. Mech. 80 (2000) 733-744.

[3] Caflish R.E., A simplified version of the abstract Cauchy-Kowalewski theorem with weak singularities, Bull. Amer. Math. Soc. 23 (1990) 495-500.

[4] Cannone M., Lombardo M.C., Sammartino M., Well-posedness of the boundary layer equations with a nonanalyticity hypothesis, (in preparation).

[5] Grenier E., On the nonlinear instability of Euler and Prandtl equations, Comm. Pure Appl. Math. 53 (2000) 10671091.

[6] Oleinik O.A., Samokhin V.N., Mathematical Models in Boundary Layer Theory, Chapman \& Hall/CRC, New York, 1999.

[7] Safonov M.V., The abstract Cauchy-Kowalewski theorem in a weighted Banach space, Comm. Pure Appl. Math. 48 (1995) 629-637.

[8] Sammartino M., Caflisch R.E., Zero viscosity limit for analytic solutions of the Navier-Stokes equation on a halfspace I. Existence for Euler and Prandtl equations, Comm. Math. Phys. 192 (1998) 433-461. 